\title{
Prospects: Reflecting back, looking forward
}

\author{
P. T. M. Marope
}

Published online: 25 September 2014

(C) UNESCO IBE 2014

I am pleased to introduce this ambitious and thought-provoking issue of Prospects, which inaugurates my appointment as the Director of the UNESCO International Bureau of Education (IBE) and Editor-in-Chief of the journal. Having served as a member of its Editorial Board for many years, I have already had the opportunity to closely witness its remarkable intellectual journey and development, and contribute actively to it. Thus, my new role of Editor-in-Chief comes as a natural next stage, one that aims at continuing to publish a journal whose high quality standards and distinctive profile, substance and identity make it a landmark among many other journals of comparative education.

It also comes with the challenge to build a stronger, even more prestigious and globally visible journal. Its merits should extend beyond publishing sound peer-reviewed academic articles, to producing well-grounded, reliable work that responds to today's educational questions and policy needs, with a special focus on curriculum-related issues. As we move toward making the IBE a global Center of Excellence, we need to reflect deeply about reorienting the journal so that it can contribute optimally to IBE's core mandate and goals; key among them is strengthening the capacity of education systems to equitably provide high quality education and effective learning opportunities.

This issue is a clear step in the new direction and illustrates our efforts to more effectively address IBE's strategic goals and mandate. Key topics featured in this issue include education quality, equity, access to learning, teacher professionalism, community participation and youth labour market integration. The geographical coverage of the articles is notable; it includes Brazil, Cambodia, Colombia, China, Ghana, Kenya, Nepal and West Africa.

The contributors to this issue are from a vast spectrum of cultures, represent different horizons of thought and belong to different generations. Despite these differences and the diversity of topics they address, the main themes of the articles are interwoven. A recurring

P. T. M. Marope $(\varangle)$

UNESCO International Bureau of Education, C.P. 199, 1211 Geneva 20, Switzerland

e-mail: m.marope@unesco.org 
line of argument throughout this issue is the need for an evidence-based participative process for improving equity of education quality and learning. More specifically, they argue that such processes should take into account the voices of all stakeholders. For instance, Steven Klees and Omar Qargha call for "widespread, highly participative debates" to answer the questions of why educators have made so little progress on equity issues and what more needs to be done. Aina Tarabini, Xavier Bonal and Oscar Valiente conclude with a caution that failure to analyse education data in context and to ensure stakeholders' participation in decision-making can lead to highly inaccurate conclusions and recommendations. Obed Mfum-Mensah and Sophia Friedson-Ridenour ask "whose voices are being heard" through diverse mechanisms for community participation in education in northern Ghana.

The articles presented in the Open File of this issue also stress the importance of the context, whether in research, policymaking, or pedagogical practice. Aina Tarabini and her colleagues emphasize that context is always important at a discursive level, but it can be neglected in practical analyses and political recommendations (see, for instance, the highly decontextualized uses of standardized test results). School for Life, a complementary education programme described by Mfum-Mensah and Friedson-Ridenour, aims at an inclusive environment that takes the local context into account and strives to provide opportunities for traditionally marginalized groups to participate in the education process. Education outcomes depend profoundly on local social, economic and cultural contexts and therefore are "much less direct and more complex than is often believed" (Colclough 2012, p. 144). This idea has repeatedly appeared in Prospects; a notable example is the Viewpoint in which Michael Crossley (2010) called for efforts to strengthen local involvement in policy-oriented research and asked that we do more to acknowledge the influence and impact of contextual factors in educational research and development cooperation. Indeed, context matters and that point is underscored by all the articles in this issue.

The issue opens with a powerful Viewpoint, co-authored by Steven Klees, a leading scholar in comparative education, and his student, Omar Qargha. They examine the case of UNICEF, which has recently decided to focus its efforts on equity in education. Based on the argument that "human rights cannot be realized so long as inequality persists" (UNICEF 2010, p. 19), the agency's shift in emphasis from a focus on human rights to one on equity is a starting point for the authors to highlight the difficulties associated with defining equity and relating it to human rights, and the need for further broad and participative discussion. They discuss five issues related to equity, which, they argue, are essential to thinking about and acting on equity in education and society: inter-agency contradictions in global policy; EFA and the MDGs; privatization and private-public partnerships (PPPs); evidence-based policy; and the right to education. Their Viewpoint will certainly contribute to a needed and widely participative debate on the topic of what equity means in education and what to do about it.

Tarabini, Bonal and Valiente discuss the complex effects generated by poverty at the school level and the variety of responses by schools situated in very similar socioeconomic and cultural contexts. Poverty is extremely heterogeneous (Dale 2010) and, in themselves, unfavourable contexts do not necessarily generate educational failure. In the authors' words, "poverty conditions but does not determine the options for developing schools". This diversity of options results from a combination of factors, including the context of poverty, the legal and institutional framework of education policy and the existing professional culture at each school. 
Mfum-Mensah and Friedson-Ridenour look at School for Life, a complementary education programme operating in northern Ghana. The programme is an important mechanism to engage and empower communities and individuals. It fits the community's social, cultural and economic context, making it very locally relevant and helping children move from the familiar to the unfamiliar. It also links the school and the community, making schooling relevant to the lived experiences of the learners. By analyzing in detail the mechanism of community participation in the programme, the authors also raise significant questions about the links between school curriculum, pedagogy and teaching in marginalized communities and students' cultural and social contexts.

Brent Edwards, Thomas Zimmermann, Chhinh Sitha, James Williams and Yuto Kitamura analyze the phenomena of student retention and dropout in Cambodia, as students transition from primary to lower secondary school. Combining complexity theoryespecially as it applies to the nexus of education and development (Mason 2009; Nordtveit 2010) — with the use of narrative research methods, their study aims to better understand the dynamics of this process and the challenges that individual families and students face around this transition. In particular, they stress the links between characteristics of the economic, education and health systems, links that make students more likely to drop out. The authors offer a few strategies to improve student retention. They argue that the cumulative effect of these strategies could have a positive impact on retention, especially if, in the terms of complexity theory, multiple systems (e.g., family, health, education and economic systems) are involved simultaneously.

Lijia Wang, Manhong Lai and Leslie Nai-Kwai Lo examine the way that performancerelated pay for teachers was recently implemented in China. Their findings reveal three main negative outcomes: compliant professionalism, a failure to improve teaching standards, and a failure to improve the quality of education. The authors highlight the need for teachers to have their voices heard about what performance should be evaluated and how. They also offer several policy recommendations regarding the performance pay reform itself: evaluations should respect and consider the complexity of teaching; qualitative approaches to evaluation should be included; and peer reviews by colleagues should be introduced, in addition to the evaluation by the principals.

Benta Abuya, Elijah Onsomu and DaKysha Moore examine the role of social construction in girls' secondary schooling in two slum schools in Nairobi. They find that the way girls are constructed in Kenyan society influences their educational experiences. These constructions-deeply rooted stereotypes that girls internalize as normative-are reinforced by several factors: the schools themselves; the interactions between home, school, community and the broader society; and the differences in magnitude of the constructions across the various environments. Their findings have significant implications for policymakers. Two key ones are the need to increase the government's grassroots campaigns, stressing the importance of a commitment to educating girls; and the need for women to take on leadership roles, to help implement change.

Conrad Hughes argues that K-12 international education can play an active role in reducing prejudice, and that it can be done effectively through curriculum design, teaching, and learning. While he points out that no panacea exists to eliminate prejudice, he shows how guiding principles in international education, mainly the International Baccalaureate (IB) and UNESCO, have successfully attempted to quell prejudice. His thorough analysis concludes that working against prejudice cannot be left to the curriculum alone or to exceptionally gifted teachers. Instead, in his words, it requires a coherent approach "at the levels of construct-domain, teaching and learning philosophy, and carefully designed programmes". 
Priyadarshani Joshi analyzes how parents' financial capacities, education levels and networks affect their schooling selection preferences, options and processes in Nepal. Despite substantial regulatory freedom to choose, most parents revealed that they had limited options. Their school choice is influenced by factors such as the increasing role that children play in schooling decision-making; the stigma around public education and the implicit growth of private schools; and the sociopolitical conditions (e.g., political conflicts, social unrest) that often lead parents to choose schools that are close to their homes. In terms of policymaking, the author describes an urgent need to focus on those children who are stuck in the lowest performing schools, by better targeting policy and resources. She also suggests making information more transparent by providing more objective quality indicators.

Christophe Jalil Nordman and Laure Pasquier-Doumer take a close look at the links between formal education and vocational training and their economic returns, in the context of seven West African countries. Their analysis is based on the 1-2-3 Surveys, conducted in West Africa in the 2000s. Their analysis indicates that vocational education could be helpful in integrating the formal sector and that, compared to general education, it often offers better earnings for the individual and helps firms improve their performance. The authors urge the design of more effective policies for poverty alleviation, as part of more efficient policies to tackle youth employment deficits in the region.

Orlando Acosta and Jorge Celis analyze the emergence of Colombian doctoral programmes within a national and international context. They show that, while Colombia has experienced significant growth in the number of graduate students per million people over the last decade, it continues to lag behind other Latin American countries. Their detailed investigation is accompanied by policy recommendations, including suggestions to stimulate the private sector's participation in science, technology and innovation activities that require highly-skilled human capital, but also to promote international cooperation to ensure student global mobility.

The articles gathered in this issue draw our attention once again to important issues that deserve more careful treatment than they have received so far. They also advocate for a stronger relationship between research, policy and practice, a relationship that is key to the success of any education initiative. All the authors in this issue conclude their articles by stating key policy implications of their research and adding recommendations for policymakers. Like many past issues of Prospects, this one illustrates the bridges between the worlds of research, policymaking and practice-an important feature of the journal and one we hope to see continue.

\section{References}

Colclough, C. (2012). Education, poverty and development: Mapping their interconnections. Comparative Education, 48(2), 135-148.

Crossley, M. (2010). Context matters in educational research and international development: Learning from the small states experience. Prospects, 40(4), 421-429.

Dale, R. (2010). The dark side of the whiteboard: Education, poverty, inequalities and social exclusion. Paper for distribution at the Belgian EU Presidency Conference on Education and Social Inclusion, September 28-29, 2010, Ghent.

Mason, M. (2009). Making educational development and change sustainable: Insights from complexity theory. International Journal of Educational Development, 29, 117-124.

Nordtveit, B. (2010). Development as a complex process of change: Conception and analysis of programs and policies. International Journal of Educational Development, 30, 110-117.

UNICEF (2010). Re-focusing on equity: Questions and answers. New York: UNICEF. 